\title{
Eficácia de Herbicidas Inibidores da ACCase no Controle de GRamíneas EM LAVOURAS DE SOJA ${ }^{1}$
}

\author{
Efficacy of ACCase-Inhibiting Herbicides in Controlling Grass Weeds in Soybean Crops
}

\author{
BARROSO, A.L.L. ${ }^{2}$, DAN, H.A. ${ }^{3}$, PROCÓPIO, S.O. ${ }^{4}$, TOLEDO, R.E.B. ${ }^{5}$, SANDANIEL, C.R. ${ }^{6}$, \\ BRAZ, G.B.P. ${ }^{6}$ e CRUVINEL, K.L. ${ }^{6}$
}

\begin{abstract}
RESUMO - Objetivou-se com este trabalho avaliar a eficácia de herbicidas inibidores da ACCase, aplicados isoladamente ou em associações, no controle das espécies de plantas daninhas pertencentes à família das gramineas Brachiaria decumbens, Digitaria ciliaris, Eleusine indica, Brachiaria plantaginea e Cenchrus echinatus, na cultura da soja. O experimento foi conduzido em campo, em delineamento de blocos ao acaso com quatro repetições. Os tratamentos avaliados foram: clethodim $\left(84 \mathrm{~g} \mathrm{ha}^{-1}\right)$, clethodim + quizalofop-p-ethyl $\left(48+40 \mathrm{~g} \mathrm{ha}^{-1}\right)$, [clethodim + fenoxaprop-p-ethyl] $\left(50+50 \mathrm{~g} \mathrm{ha}^{-1}\right)$, sethoxydim $\left(230 \mathrm{~g} \mathrm{ha}^{-1}\right)$, tepraloxydim (100 $\left.\mathrm{g} \mathrm{ha}^{-1}\right)$, fluazifop-p-butyl (125 $\left.\mathrm{g} \mathrm{ha}^{-1}\right)$, haloxyfop-methyl (60 $\left.\mathrm{g} \mathrm{ha}^{-1}\right) \mathrm{e}$ testemunha sem herbicida. A convivência das plantas de soja com as gramineas infestantes resultou em perda significativa na produtividade de grãos. Os melhores niveis de controle de $B$. decumbens foram verificados com a utilização de haloxyfop-methyl. Tepraloxydim pode ser considerado seletivo a $B$. decumbens. Nenhum tratamento proporcionou controle final de D. ciliaris superior a $90 \%$, porém menor eficiência foi verificada quando se aplicaram sethoxydim e fluazifop-p-butyl. Apenas os tratamentos sethoxydim e [clethodim + fenoxaprop-p-ethyl] não mostraram controle satisfatório de $E$. indica. $B$. plantaginea foi a espécie mais facilmente controlada pelos herbicidas avaliados; no entanto, haloxyfop-methyl, tepraloxydim, clethodim e [clethodim + fenoxaprop-p-ethyl] se destacaram no controle dessa invasora. A adição de quizalofop-p-ethyl ao clethodim proporcionou incremento significativo no controle de C. echinatus. Também os herbicidas haloxyfop-methyl e tepraloxydim apresentaram controle satisfatório dessa espécie daninha.
\end{abstract}

Palavras-chave: Brachiaria decumbens, Digitaria ciliaris, Eleusine indica, Brachiaria plantaginea e Cenchrus echinatus.

\begin{abstract}
The objective of this work was to evaluate the efficacy of ACCase-inhibitors (ariloxyfenoxypropionates and cyclohexanodiones), applied alone or in combination, in controlling the grass weed species Brachiaria decumbens, Digitaria ciliaris, Eleusine indica, Brachiaria plantaginea and Cenchrus echinatus in soybean crop. The study was carried out in the field in a randomized block design with four replicates. The following treatments were evaluated: clethodim $\left(84 \mathrm{~g} \mathrm{ha}^{-1}\right)$, clethodim + quizalofop-p-ethyl $\left(48+40 \mathrm{~g} \mathrm{ha}^{-1}\right)$, [clethodim + fenoxaprop-p-ethyl] $\left(50+50 \mathrm{~g} \mathrm{ha}^{-1}\right)$, sethoxydim $\left(230 \mathrm{~g}\right.$ ha-1), tepraloxydim $\left(100 \mathrm{~g} \mathrm{ha}{ }^{-1}\right)$, fluazifop-p-butyl $\left(125 \mathrm{~g} \mathrm{ha} \mathrm{w}^{-1}\right)$, haloxyfop-methyl $\left(60 \mathrm{~g} \mathrm{ha}^{-1}\right)$ and control (no herbicide). In the presence of the infesting weeds, soybean grain yield was significantly reduced. The highest efficiency of $\boldsymbol{B}$. decumbens control was observed with the application of haloxyfop-methyl. Tepraloxydim was quite selective to B. decumbens. No treatment promoted a final control of $\boldsymbol{D}$. ciliaris higher than $90 \%$; nevertheless, the lowest efficiencies were verified with the application of sethoxydim and fluazifop-p-butyl. The only treatments that did not present a satisfactory control of $\boldsymbol{E}$. indica were sethoxydim and [clethodim + fenoxaprop-p-ethyl]. The species most easily controlled by the herbicides evaluated
\end{abstract}

Recebido para publicação em 27.6.2009 e na forma revisada em 12.3.2010.

2 Professor da Faculdade de Agronomia da Fesurv, Universidade de Rio Verde, 75901-970 Rio Verde-GO; ${ }^{3}$ Mestrando do Programa de Pós-Graduação da Fesurv - Universidade de Rio Verde; ${ }^{4}$ Pesquisador da Embrapa Tabuleiros Costeiros, $49025-040$ Aracaju-SE, <procopio@cpatc.embrapa.br>, Bolsista do CNPq; ${ }^{5}$ Eng $^{0}-$ Agr $^{\circ}$., D.Sc., 13405-367 Piracicaba-SP; ${ }^{6}$ Discente de Agronomia da Fesurv - Universidade de Rio Verde. 
was B. plantaginea. However, haloxyfop-methyl, tepraloxydim, clethodim and /clethodim + fenoxaprop$p$-ethyl] presented the highest efficiency rates for controlling this weed. The addition of quizalofop$p$-ethyl to clethodim significantly increased $C$. echinatus control The herbicides haloxyfop-methyl and tepraloxydim also presented a satisfactory control of this grass weed.

Keywords: Brachiaria decumbens, Digitaria ciliaris, Eleusine indica, Brachiaria plantaginea and Cenchrus echinatus.

\section{INTRODUÇÃO}

A competição de plantas daninhas com a cultura da soja (Glycine max) pode se refletir em perdas relevantes na produtividade de grãos (Silva et al., 2009). As plantas daninhas competem com as plantas de soja por recursos como luz, água e nutrientes, além de hospedarem organismos muitas vezes indesejáveis (Silva et al., 1999).

Das plantas daninhas com alto potencial competitivo com a cultura da soja, podem-se destacar as espécies pertencentes à família das gramineas. De acordo com Barros et al. (1992), as gramineas têm provocado maiores danos à cultura da soja, quando comparadas com as dicotiledôneas. Fleck (1995) verificou reduções no rendimento de grãos na cultura da soja de 18 a $82 \%$ devido à interferência causada pela presença de diferentes densidades de Brachiaria plantaginea.

O controle de plantas daninhas gramíneas é realizado nas culturas dicotiledôneas - como soja, por exemplo - predominantemente com herbicidas inibidores da enzima acetil-CoA carboxilase (ACCase). Estes promovem a inibição enzimática, bloqueando a síntese de lipídeos nas plantas suscetiveis (Burke et al., 2006), prejudicando a formação das paredes celulares e desestruturando os tecidos em formação (Nalewaja et al., 1994). Os principais sintomas promovidos pela ação desses herbicidas são: paralisação do crescimento, amarelecimento das folhas, coloração arroxeada ou avermelhada nas folhas mais velhas, seguida de morte apical (DeFelice et al., 1989).

Os herbicidas pertencentes a essa família (ACCase) estão distribuídos em três grupos químicos: os ariloxyfenoxypropionatos (APP), os cicloexanodionas (CHD) e os phenylpyrazolines (Hochberg et al., 2009). Os herbicidas inibidores de ACCase compõem uma das classes mais numerosas de herbicidas registrados no Brasil (Vidal, 2002).

Por mais que os herbicidas inibidores da ACCase tenham o mesmo mecanismo de ação, sabe-se que esses produtos apresentam diferenças quanto ao espectro de ação no controle de gramineas (Harwood, 1999). López-Ovejero et al. (2006) comentam que, apesar de os herbicidas inibidores da ACCase apresentarem o mesmo mecanismo de ação, existem diferenças marcantes entre os diferentes grupos químicos. Lingenfelter \& Curran (2007) constataram que os herbicidas fluazifop-p-butyl e clethodim foram mais eficientes no controle de Muhlenbergia frondosa em relação a quizalofop-p-ethyl e sethoxydim. Askew et al. (2000) observaram que clethodim, fluazifopp-butyl, quizalofop-p-ethyl e sethoxydim não foram eficientes no controle do arroz-vermelho. Marshall et al. (1994) verificaram diferenças de controle entre os herbicidas inibidores de ACCase: os aryloxyphenoxypropionatos apresentaram menor eficiência no controle de $E$. indica em relação aos cicloexanodionas.

São poucas as informações referentes ao desempenho dos herbicidas inibidores de ACCase no controle das principais gramineas infestantes das lavouras de soja na região dos cerrados. Esse desconhecimento gera informações errôneas de que o espectro de ação e a eficácia desses herbicidas são muito semelhantes entre os herbicidas que possuem esse mecanismo de ação, o que faz com que o aspecto econômico seja em várias situações o único critério a ser levado em consideração na definição do produto a ser adquirido. Também, ressalta-se um outro entrave na melhoria do processo de escolha correta do graminicida: a dificuldade de identificação das gramineas infestantes nos primeiros estádios de desenvolvimento. Sem uma identificação 
correta dessas espécies, não é possível optar por um determinado herbicida que tenha um espectro de ação mais adequado a determinadas comunidades infestantes.

Tendo em vista o cenário exposto, objetivou-se com este trabalho avaliar a eficácia de herbicidas inibidores da ACCase (ariloxyfenoxypropionatos e cicloexanodionas), aplicados isoladamente ou em associações, no controle de cinco espécies de plantas daninhas pertencentes à família das gramíneas, na cultura da soja.

\section{MATERIAL E MÉTODOS}

O experimento foi conduzido durante a safra 2006/2007, no municipio de Rio VerdeGO, cujas coordenadas geográficas são: latitude de $17^{\circ} 47^{\prime} 24^{\prime \prime} \mathrm{S}$, longitude de $50^{\circ} 56^{\prime} 31^{\prime \prime} \mathrm{W}$ e 698 metros acima do nivel do mar. O clima regional, classificado pelo sistema internacional de Köppen, é do tipo CWA, úmido e inverno seco, com precipitação média anual de $1.500 \mathrm{~mm}$ e temperatura média anual de $25{ }^{\circ} \mathrm{C}$.

O solo da área experimental é classificado como Latossolo Vermelho distrófico, possuindo $450 \mathrm{~g} \mathrm{~kg}^{-1}$ de argila, $60 \mathrm{~g} \mathrm{~kg}^{-1}$ de silte, $490 \mathrm{~g} \mathrm{~kg}^{-1}$ de areia, com uma saturação por bases de $53 \%$. Antes da semeadura, o solo foi preparado convencionalmente, com grade aradora e duas operações com grade niveladora.

O cultivar de soja Conquista foi semeado num espaçamento de $0,5 \mathrm{~m}$ entre as linhas, com uma população de 350 mil plantas por hectare. Junto da semeadura, foi realizada uma adubação de base, com $400 \mathrm{~kg} \mathrm{ha}^{-1}$ da fórmula comercial N-P-K 2-20-18. Após o plantio da soja, procedeu-se à semeadura das gramineas infestantes (Brachiaria decumbens, Digitaria ciliaris, Eleusine indica, Brachiaria plantaginea e Cenchrus echinatus) nas entrelinhas da cultura; em cada entrelinha semeouse uma espécie infestante diferente. Esse procedimento visou apenas induzir a emergência das espécies infestantes de interesse investigativo de uma forma a evitar erros na identificação dessas gramíneas em estádios iniciais, além de facilitar a eliminação de outras espécies emergidas a partir do banco natural de sementes do solo.
O delineamento experimental utilizado foi o de blocos ao acaso, composto de oito tratamentos com quatro repetições. Os tratamentos foram: clethodim ${ }^{(1)}\left(84 \mathrm{~g} \mathrm{ha}^{-1}\right)$, clethodim + quizalofop-p-ethyl ${ }^{(1)}\left(48+40 \mathrm{~g} \mathrm{ha}^{-1}\right)$, [clethodim + fenoxaprop-p-ethyl] $\left(50+50 \mathrm{~g} \mathrm{ha}^{-1}\right)$, sethoxydim $^{(2)}$ (230 $\left.\mathrm{g} \mathrm{ha}^{-1}\right)$, tepraloxydim ${ }^{(3)}$ (100 $\left.\mathrm{g} \mathrm{ha}^{-1}\right)$, fluazifop-p-butyl (125 $\mathrm{g} \mathrm{ha}^{-1}$ ), haloxyfop-methyl ${ }^{(2)}\left(60 \mathrm{~g} \mathrm{ha}^{-1}\right)$ e testemunha sem herbicida. Cada parcela foi alocada em uma área de $18 \mathrm{~m}^{2}$ (3 x $\left.6 \mathrm{~m}\right)$.

A aplicação dos tratamentos herbicidas foi realizada 23 dias após a emergência da soja (estádio V4), utilizando um pulverizador costal de precisão com pressurização por $\mathrm{CO}_{2}$, equipado com barra contendo seis pontas de pulverização do tipo TT 110-02, calibrado para um volume de calda equivalente a $200 \mathrm{~L} \mathrm{ha}^{-1}$. As condições ambientais no momento da aplicação eram as seguintes: temperatura minima de $26{ }^{\circ} \mathrm{C}$, média de $29,1{ }^{\circ} \mathrm{C}$ e máxima de $31,1{ }^{\circ} \mathrm{C}$; UR mínima de $49 \%$, média de $51 \%$ e máxima de $63 \%$; e velocidade média do vento de $6 \mathrm{~km} \mathrm{~h}^{-1}$.

Por ocasião da aplicação dos herbicidas, a densidade e o estádio de desenvolvimento das gramineas eram: $B$. decumbens -15 plantas por metro, com um a dois perfilhos por planta; D. ciliaris -15 plantas por metro, com um a três perfilhos por planta; $E$. indica - nove plantas por metro, com dois a quatro perfilhos por planta; B. plantaginea - 12 plantas por metro, com um a três perfilhos por planta; $C$. echinatus - 10 plantas por metro, com um a três perfilhos por planta. Durante a condução do experimento, as demais plantas daninhas que porventura emergiam nas parcelas foram erradicadas quinzenalmente por meio de arranque manual.

As avaliações de controle das plantas daninhas foram feitas de forma visual aos 14, 21, 29 e 44 dias após a aplicação dos tratamentos (DAA), utilizando-se escala percentual de 0 (zero) a $100 \%$, em que 0 (zero) representa ausência de sintomas e $100 \%$, morte de todas as plantas. A produtividade da cultura foi obtida através da colheita manual das unidades experimentais (quatro linhas centrais, descontando-se $0,5 \mathrm{~m}$ de cada extremidade).

(1) Adicionado 1,0 L ha-1 do adjuvante Lanzar ${ }^{\circledR}$. (2) Adicionado 1,0 L ha' ${ }^{-1}$ do adjuvante Assist ${ }^{\circledR}$. (3) Adicionado 1,0 L ha-1 do adjuvante Dash ${ }^{\circledR}$. 
A produtividade foi estimada após a correção da umidade dos grãos para $13 \%$.

Os dados foram submetidos à análise de variância, e as médias das variáveis significativas, agrupadas pelo critério de Scott Knott a $5 \%$ de significância.

\section{RESULTADOS E DISCUSSÃO}

Todos os tratamentos herbicidas mostraram baixos niveis de controle de Brachiaria decumbens aos 14 dias após a aplicação (DAA), porém os tratamentos sethoxydim, [clethodim + fenoxaprop-p-ethyl] e haloxyfop-methyl apresentaram superioridade em relação aos demais (Tabela 1). Aos 21 DAA, observou-se que o herbicida haloxyfop-methyl apresentou o maior nível de controle dessa espécie infestante $(82 \%)$, que foi evoluindo nas demais avaliações, atingindo $96 \%$ na última, realizada aos 44 DAA. Em contrapartida, o herbicida tepraloxydim teve o pior desempenho no controle de $B$. decumbens. Essa espécie foi se recuperando dos efeitos iniciais do herbicida, chegando ao ponto de ser constatado controle inferior a $10 \%$ aos 44 DAA (Tabela 1). Esse comportamento de "seletividade" do tepraloxydim a $B$. decumbens pode ser explorado futuramente, no intuito de esse herbicida ser aplicado em áreas de cultivo dessa forrageira para o controle de outras gramíneas infestantes. Essa opção pode ser extremamente interessante devido à elevada extensão de áreas de pastagens formadas com
B. decumbens, em diversas regiões do Brasil; por não existir até o momento nenhuma opção para o controle químico de outras gramineas nessas áreas, tem-se apenas a opção de herbicidas para o controle de latifoliadas. A associação entre quizalofop-p-ethyl e clethodim ou mesmo a aplicação da mistura pré-formulada [clethodim + fenoxaprop-p-ethyl] não proporcionaram incrementos significativos no controle de $B$. decumbens, em relação à utilização isolada de clethodim. Todos esses tratamentos mostraram baixa eficácia no controle dessa graminea, sendo essa falta de eficiência também verificada com a aplicação do sethoxydim. Fluazifop-p-butyl apresentou controle final razoável de $B$. decumbens $(72 \%$ aos $44 \mathrm{DAA})$ (Tabela 1), porém ainda bem inferior ao desempenho do haloxyfop-methyl, que se mostrou a melhor opção em áreas com alta infestação dessa invasora.

Na avaliação da sensibilidade de Digitaria ciliares perante a aplicação dos herbicidas (Tabela 2), verificou-se que aos 14 DAA os niveis de controle oscilaram entre 32 e $53 \%$, com destaque para os tratamentos clethodim, clethodim + quizalofop-p-ethyl, tepraloxydim, [clethodim + fenoxaprop-p-ethyl] e haloxyfopmethyl. Esses cinco tratamentos continuaram apresentando os melhores niveis de controle nas demais avaliações $(21,29$ e 44 DAA); contudo, nenhum desses tratamentos resultou em controle de $D$. ciliares acima de $90 \%$ aos 44 DAA. Esses resultados são preocupantes, principalmente quando se observam aumentos

Tabela 1 - Controle de capim-braquiária (Brachiaria decumbens) por diferentes tratamentos herbicidas aplicados em pós-emergência. Rio Verde-GO. 2006/2007

\begin{tabular}{|c|c|c|c|c|c|}
\hline \multirow{2}{*}{ Tratamento } & \multirow{2}{*}{$\begin{array}{c}\text { Dose } \\
(\text { g i.a. })^{1 /} \text { ou }(\text { e.a. })^{2 /} \mathrm{ha}^{-1}\end{array}$} & \multicolumn{4}{|c|}{ Controle $(\%)$} \\
\hline & & $14 \mathrm{DAA}^{3 /}$ & $21 \mathrm{DAA}$ & $29 \mathrm{DAA}$ & 44 DAA \\
\hline clethodim & 84 & $37 \mathrm{~b}$ & $57 \mathrm{c}$ & $45 \mathrm{c}$ & $33 \mathrm{c}$ \\
\hline clethodim + quizalofop-p-ethyl & $48+40$ & $40 \mathrm{~b}$ & $61 \mathrm{c}$ & $51 \mathrm{c}$ & $38 \mathrm{c}$ \\
\hline [clethodim + fenoxaprop-p-ethyl] & {$[50+50]$} & $45 \mathrm{a}$ & $68 \mathrm{~b}$ & $67 \mathrm{~b}$ & $41 \mathrm{c}$ \\
\hline sethoxydim & 230 & $46 \mathrm{a}$ & $68 \mathrm{~b}$ & $56 \mathrm{~b}$ & $43 \mathrm{c}$ \\
\hline tepraloxydim & 100 & $38 \mathrm{~b}$ & $28 \mathrm{~d}$ & $18 \mathrm{~d}$ & $9 \mathrm{~d}$ \\
\hline fluazifop-p-butyl & 125 & $36 \mathrm{~b}$ & $65 \mathrm{~b}$ & $82 \mathrm{a}$ & $72 \mathrm{~b}$ \\
\hline haloxyfop-methyl & 60 & $44 \mathrm{a}$ & $82 \mathrm{a}$ & $94 \mathrm{a}$ & $96 \mathrm{a}$ \\
\hline testemunha sem herbicida & - & $0 \mathrm{c}$ & $0 \mathrm{e}$ & $0 \mathrm{e}$ & $0 \mathrm{~d}$ \\
\hline \multicolumn{2}{|c|}{$\mathrm{CV}(\%)$} & 10,5 & 12,1 & 13,5 & 8,3 \\
\hline
\end{tabular}

Médias seguidas de mesma letra, na coluna, não diferem entre si pelo teste de Scott Knott a 5\%; ${ }^{1 /}$ Ingrediente ativo; ${ }^{2 /}$ Equivalente ácido, utilizado para o herbicida haloxyfop-methyl; ${ }^{3 /}$ Dias após a aplicação. 
Tabela 2 - Controle de capim-colchão (Digitaria ciliaris) por diferentes tratamentos herbicidas aplicados em pós-emergência. Rio Verde-GO. 2006/2007

\begin{tabular}{|c|c|c|c|c|c|}
\hline \multirow{2}{*}{ Tratamento } & \multirow{2}{*}{$\begin{array}{c}\text { Dose } \\
(\text { g i.a. })^{1 /} \text { ou }(\text { e.a. })^{2 / /} \mathrm{ha}^{-1}\end{array}$} & \multicolumn{4}{|c|}{ Controle (\%) } \\
\hline & & $14 \mathrm{DAA}^{3 /}$ & $21 \mathrm{DAA}$ & 29 DAA & 44 DAA \\
\hline clethodim & 84 & $53 \mathrm{a}$ & $81 \mathrm{a}$ & $90 \mathrm{a}$ & $79 \mathrm{a}$ \\
\hline clethodim + quizalofop-p-ethyl & $48+40$ & $53 \mathrm{a}$ & $78 \mathrm{a}$ & $87 \mathrm{a}$ & $74 \mathrm{a}$ \\
\hline [clethodim + fenoxaprop-p-ethyl] & {$[50+50]$} & $48 \mathrm{a}$ & $76 \mathrm{a}$ & $84 \mathrm{a}$ & $76 \mathrm{a}$ \\
\hline sethoxydim & 230 & $44 \mathrm{~b}$ & $67 \mathrm{~b}$ & $76 \mathrm{~b}$ & $54 \mathrm{~b}$ \\
\hline tepraloxydim & 100 & $53 \mathrm{a}$ & $79 \mathrm{a}$ & $91 \mathrm{a}$ & $84 \mathrm{a}$ \\
\hline fluazifop-p-butyl & 125 & $32 \mathrm{c}$ & $70 \mathrm{~b}$ & $78 \mathrm{~b}$ & $60 \mathrm{~b}$ \\
\hline haloxyfop-methyl & 60 & $48 \mathrm{a}$ & $82 \mathrm{a}$ & $90 \mathrm{a}$ & $84 \mathrm{a}$ \\
\hline testemunha sem herbicida & - & $0 \mathrm{~d}$ & $0 \mathrm{c}$ & $0 \mathrm{c}$ & $0 \mathrm{c}$ \\
\hline \multicolumn{2}{|c|}{$\mathrm{CV}(\%)$} & 10,2 & 9,9 & 9,7 & 7,4 \\
\hline
\end{tabular}

Médias seguidas de mesma letra, na coluna, não diferem entre si pelo teste de Scott Knott a 5\%; ${ }^{1 /}$ Ingrediente ativo; ${ }^{2 /}$ Equivalente ácido, utilizado para o herbicida haloxyfop-methyl; ${ }^{3 /}$ Dias após a aplicação.

relevantes da infestação dessa espécie nas lavouras de soja cultivadas na região do cerrado. Sethoxydim e fluazifop-p-butyl, nas doses avaliadas, que estão dentro da faixa de recomendação desses herbicidas, mostraram desempenho insatisfatório no controle de D. ciliares: 54 e $60 \%$, respectivamente, aos 44 DAA (Tabela 2). Gelmine et al. (2001) constataram que o uso de clethodim de forma isolada proporcionou controle superior a $90 \%$ de $D$. horizontalis. Em áreas cultivadas com soja convencional que apresentem alta infestação de $D$. ciliaris, uma opção a ser estudada seria a utilização de doses mais elevadas de um dos cinco tratamentos que apresentaram maior desempenho no presente experimento (haloxyfop-methyl, tepraloxydim, clethodim, [clethodim + fenoxaprop-p-ethyl] e clethodim + quizalofop-p-ethyl). Também, a rotação da área com soja tolerante a glyphosate pode ser uma alternativa interessante para a diminuição do banco de sementes de $D$. ciliaris, pois o glyphosate é considerado o melhor graminicida de aplicação em pós-emergência disponivel na atualidade. A inserção, seja em tanque do quizalofop-p-ethyl ou do fenoxapropp-ethyl na própria formulação junto ao clethodim, não proporcionou aumento no controle de $D$. ciliares, em comparação à aplicação isolada de clethodim.

Os tratamentos herbicidas sethoxydim e [clethodim + fenoxaprop-p-ethyl] mostraram os piores desempenhos no controle de Eleusine indica, fato evidenciado, sobretudo, nas três últimas avaliações (Tabela 3), indicando não serem recomendados para o controle dessa invasora. Os demais tratamentos mostraram eficácia semelhante no controle de $E$. indica; clethodim + quizalofop-p-ethyl e haloxyfopmethyl mantiveram controle acima de $90 \%$ até a última avaliação (44 DAA). Esses dados revelam que a adição de quizalofop-p-ethyl ao clethodim ocasionou leve melhora no controle de E. indica, ao contrário da presença do fenoxaprop-p-ethyl, que acarretou redução no nivel de controle em relação à aplicação isolada de clethodim. Esses resultados contradizem parcialmente os obtidos por Marshall et al. (1994), os quais concluiram que herbicidas pertencentes à família dos aryloxyphenoxypropionatos apresentaram niveis inferiores de controle de E. indica, quando comparados com os cicloexanodionas. Vidal et al. (2006) constataram controle eficiente de um biótipo de $E$. indica originário do Estado do Mato Grosso com a aplicação dos herbicidas clethodim, quizalofop-p-ethyl, haloxyfop-methyl e fluazifop-p-butyl, porém observaram controle ineficiente quando foram utilizados os herbicidas fenoxaprop-p-ethyl e sethoxydim. Um fato a ser destacado é que, entre as espécies infestantes avaliadas, as plantas de E. indica encontravam-se, em média, com dois a quatro perfilhos, o que já não caracteriza mais uma aplicação em pós-emergência inicial. Esse fato pode ter causado diminuição generalizada na eficiência de todos os tratamentos herbicidas avaliados. Pesquisas mostram que o 
controle de gramíneas é mais eficiente quando os indivíduos estão em estádio de crescimento variando de plantas recém-emergidas até a emissão do primeiro perfilho (Chernicky et al., 1984; Deer et al., 1985).

Com relação ao controle de Brachiaria plantaginea, observou-se na Tabela 4 que aos 14 DAA nenhum dos tratamentos obteve controle superior a $80 \%$, porém ele foi superior à testemunha sem aplicação. Todavia, na avaliação seguinte (21 DAA), clethodim, haloxyfop-methyl e clethodim + quizalofop-pethyl já proporcionaram controle igual ou superior a $80 \%$. Os níveis de controle de $B$. plantaginea de todos os tratamentos continuaram evoluindo até os 29 DAA. No entanto, na avaliação final (44 DAA) apenas os tratamentos haloxyfop-methyl e tepraloxydim mantiveram o controle dessa espécie acima de $90 \%$, mas não diferindo dos resultados obtidos quando foram aplicados os tratamentos clethodim e [clethodim + fenoxaprop-p-ethyl] (Tabela 4). Fleck et al. (1997) relatam que o clethodim pode controlar $B$. plantaginea de maneira eficiente desde que esse herbicida seja aplicado quando as plantas estiverem com no máximo três perfilhos. Os demais tratamentos não finalizaram o ciclo de avaliações com resultados satisfatórios no controle de B. plantaginea. Foi constatado que nenhum dos princípios ativos associados ao clethodim (quizalofop-p-ethyl ou fenoxaprop-p-ethyl) agregou em eficácia no combate a

Tabela 3 - Controle de capim-pé-de-galinha (Eleusine indica) por diferentes tratamentos herbicidas. Rio Verde-GO. 2006/2007

\begin{tabular}{|c|c|c|c|c|c|}
\hline \multirow{2}{*}{ Tratamento } & \multirow{2}{*}{$\begin{array}{c}\text { Dose } \\
\frac{(\text { g i.a. })^{1 /} \text { ou }(\text { e.a. })^{2 / /} \mathrm{ha}^{-1}}{}\end{array}$} & \multicolumn{4}{|c|}{ Controle $(\%)$} \\
\hline & & $14 \mathrm{DAA}^{3 /}$ & $21 \mathrm{DAA}$ & $29 \mathrm{DAA}$ & $44 \mathrm{DAA}$ \\
\hline clethodim & 84 & $73 \mathrm{a}$ & $90 \mathrm{a}$ & $91 \mathrm{a}$ & $81 \mathrm{a}$ \\
\hline clethodim + quizalofop-p-ethyl & $48+40$ & $76 \mathrm{a}$ & $90 \mathrm{a}$ & $93 \mathrm{a}$ & $93 \mathrm{a}$ \\
\hline [clethodim + fenoxaprop-p-ethyl] & {$[50+50]$} & $69 \mathrm{a}$ & $79 \mathrm{~b}$ & $82 \mathrm{~b}$ & $70 \mathrm{~b}$ \\
\hline sethoxydim & 230 & $57 \mathrm{~b}$ & $74 \mathrm{~b}$ & $73 \mathrm{~b}$ & $57 \mathrm{~b}$ \\
\hline tepraloxydim & 100 & $74 \mathrm{a}$ & $89 \mathrm{a}$ & $93 \mathrm{a}$ & $88 \mathrm{a}$ \\
\hline fluazifop-p-butyl & 125 & $69 \mathrm{a}$ & $92 \mathrm{a}$ & $95 \mathrm{a}$ & $85 \mathrm{a}$ \\
\hline haloxyfop-methyl & 60 & $67 \mathrm{a}$ & $91 \mathrm{a}$ & $94 \mathrm{a}$ & $92 \mathrm{a}$ \\
\hline testemunha sem herbicida & - & $0 \mathrm{c}$ & $0 \mathrm{c}$ & $0 \mathrm{c}$ & $0 \mathrm{c}$ \\
\hline \multicolumn{2}{|c|}{$\mathrm{CV}(\%)$} & 11,4 & 9,4 & 10,0 & 13,4 \\
\hline
\end{tabular}

Médias seguidas de mesma letra, na coluna, não diferem entre si pelo teste de Scott Knott a 5\%; ${ }^{1 /}$ Ingrediente ativo; ${ }^{2 /}$ Equivalente ácido, utilizado para o herbicida haloxyfop-methyl; ${ }^{3 /}$ Dias após a aplicação.

Tabela 4 - Controle de capim-marmelada (Brachiaria plantaginea) por diferentes tratamentos herbicidas. Rio Verde-GO. 2006/2007

\begin{tabular}{|c|c|c|c|c|c|}
\hline \multirow{2}{*}{ Tratamento } & \multirow{2}{*}{ 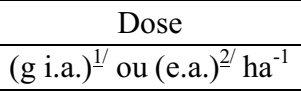 } & \multicolumn{4}{|c|}{ Controle $(\%)$} \\
\hline & & $14 \mathrm{DAA}^{3 /}$ & $21 \mathrm{DAA}$ & 29 DAA & 44 DAA \\
\hline clethodim & 84 & $72 \mathrm{a}$ & $83 \mathrm{a}$ & $92 \mathrm{a}$ & $87 \mathrm{a}$ \\
\hline clethodim + quizalofop-p-ethyl & $48+40$ & $70 \mathrm{a}$ & $80 \mathrm{a}$ & $87 \mathrm{~b}$ & $77 \mathrm{~b}$ \\
\hline [clethodim + fenoxaprop-p-ethyl] & {$[50+50]$} & $69 \mathrm{a}$ & $79 \mathrm{~b}$ & $90 \mathrm{a}$ & $84 \mathrm{a}$ \\
\hline sethoxydim & 230 & $68 \mathrm{a}$ & $78 \mathrm{~b}$ & $85 \mathrm{~b}$ & $73 \mathrm{~b}$ \\
\hline tepraloxydim & 100 & $70 \mathrm{a}$ & $79 \mathrm{~b}$ & $91 \mathrm{a}$ & $92 \mathrm{a}$ \\
\hline fluazifop-p-butyl & 125 & $64 \mathrm{~b}$ & $77 \mathrm{~b}$ & $88 \mathrm{~b}$ & $77 \mathrm{~b}$ \\
\hline haloxyfop-methyl & 60 & $68 \mathrm{a}$ & $82 \mathrm{a}$ & $92 \mathrm{a}$ & $93 \mathrm{a}$ \\
\hline testemunha sem herbicida & - & $0 \mathrm{c}$ & $0 \mathrm{c}$ & $0 \mathrm{c}$ & $0 \mathrm{c}$ \\
\hline \multicolumn{2}{|c|}{$\mathrm{CV}(\%)$} & 10,3 & 11,2 & 8,1 & 15,3 \\
\hline
\end{tabular}

Médias seguidas de mesma letra, na coluna, não diferem entre si pelo teste de Scott Knott a 5\%; ${ }^{1 /}$ Ingrediente ativo; ${ }^{2 /}$ Equivalente ácido, utilizado para o herbicida haloxyfop-methyl; ${ }^{3 /}$ Dias após a aplicação. 
B. plantaginea. Os resultados obtidos por Christoffoleti et al. (2001) foram levemente superiores aos encontrados no presente ensaio. Gazziero et al. (2000) não observaram diferenças marcantes entre os herbicidas clethodim, haloxyfop-methyl, sethoxydim e fluazifop-p-butyl no controle de $B$. plantaginea.

Fluazifop-p-butyl e sethoxydim apresentaram o menor nivel de controle de Cenchrus echinatus aos 14 DAA (Tabela 5). Sete dias após essa avaliação, o único tratamento que promoveu controle acima de $80 \%$ dessa invasora foi clethodim + quizalofop-p-ethyl, mostrando ser aquele que apresenta maior velocidade de ação sobre C. echinatus. Aos 29 DAA, além da associação de clethodim com quizalofop-pethyl, mais dois tratamentos começaram a se destacar no controle de C. echinatus: haloxyfopmethyl e tepraloxydim - todos já apresentando controle acima de $90 \%$. Esse nível de controle verificado quando da aplicação desses três tratamentos manteve-se na última avaliação (44 DAA), onde se observa que a combinação de clethodim com quizalofop-p-ethyl acarretou controle médio de C. echinatus de 99\%, ou seja, quase todas as plantas, de todas as repetições, se encontravam mortas nesse período. Para Cruz et al. (1991), tanto o fluazifop-p-butyl quanto o haloxyfop-methyl foram eficientes no controle de C. echinatus aos 28 DAA. Medrano et al. (1997) afirmam que os herbicidas haloxyfop-methyl e fluazifop-p-butyl controlaram satisfatoriamente C. echinatus. Esses resultados corroboram os encontrados no presente trabalho, principalmente em relação ao haloxyfop-methyl. De todas as gramineas avaliadas, a melhor resposta da adição de um outro princípio ativo ao clethodim - no caso, o quizalofop-p-ethyl - ocorreu no controle de C. echinatus. Essa adição resultou, aos 44 DAA, em aumento de $33 \%$ no controle dessa invasora (Tabela 5), mostrando que essa opção pode ser uma estratégia de grande valia em áreas com alta infestação de $C$. echinatus, as quais já são extremamente comuns em áreas de produção de soja da região do cerrado.

A convivência das plantas de soja com as gramíneas infestantes resultou em perda significativa na produtividade de grãos (Tabela 6). Fleck et al. (1997) observaram que a aplicação de clethodim, além de proporcionar controle satisfatório de $B$. plantaginea, também resultou em ganhos na produtividade da cultura da soja. No tratamento em que não se efetuou o controle das plantas daninhas a produtividade média da soja foi de $1.305 \mathrm{~kg} \mathrm{ha}^{-1}$, muito inferior em relação à média dos tratamentos em que foram aplicados herbicidas (2.334 kg ha $\mathrm{k}^{-1}$ ) (Tabela 6). Todavia, não se constataram diferenças significativas na produtividade de grãos de soja entre os tratamentos herbicidas, mostrando que as diferenças nos níveis de controle das gramíneas infestantes não se refletiram na produtividade da cultura. Trabalhos mostraram que o controle total de plantas daninhas não é necessário para a expressão do máximo potencial produtivo da soja (Hagood Junior et al., 1980; DeFelice et al., 1989).

Tabela 5 - Controle de timbete (Cenchrus echinatus) por diferentes tratamentos herbicidas. Rio Verde-GO. 2006/2007

\begin{tabular}{|c|c|c|c|c|c|}
\hline \multirow{2}{*}{ Tratamento } & Dose & \multicolumn{4}{|c|}{ Controle $(\%)$} \\
\hline & $\left(\text { g i.a. }^{1 /} \text { ou (e.a. }\right)^{2 /} \mathrm{ha}^{-1}$ & $14 \mathrm{DAA}^{3 /}$ & $21 \mathrm{DAA}$ & 29 DAA & 44 DAA \\
\hline clethodim & 84 & $65 \mathrm{a}$ & $73 \mathrm{c}$ & $80 \mathrm{c}$ & $66 \mathrm{c}$ \\
\hline clethodim + quizalofop-p-ethyl & $48+40$ & $72 \mathrm{a}$ & $86 \mathrm{a}$ & $95 \mathrm{a}$ & $99 \mathrm{a}$ \\
\hline [clethodim + fenoxaprop-p-ethyl] & {$[50+50]$} & $68 \mathrm{a}$ & $76 \mathrm{c}$ & $87 \mathrm{~b}$ & $82 \mathrm{~b}$ \\
\hline sethoxydim & 230 & $59 \mathrm{~b}$ & $68 \mathrm{c}$ & $74 \mathrm{c}$ & $56 \mathrm{~d}$ \\
\hline tepraloxydim & 100 & $71 \mathrm{a}$ & $78 \mathrm{~b}$ & $92 \mathrm{a}$ & $91 \mathrm{a}$ \\
\hline fluazifop-p-butyl & 125 & $55 \mathrm{~b}$ & $74 \mathrm{c}$ & $87 \mathrm{~b}$ & $84 \mathrm{~b}$ \\
\hline haloxyfop-methyl & 60 & $66 \mathrm{a}$ & $79 \mathrm{~b}$ & $93 \mathrm{a}$ & $94 \mathrm{a}$ \\
\hline testemunha sem herbicida & - & $0 \mathrm{c}$ & $0 \mathrm{~d}$ & $0 \mathrm{~d}$ & $0 \mathrm{e}$ \\
\hline \multicolumn{2}{|c|}{ CV $(\%)$} & 9,4 & 8,2 & 7,4 & 12,4 \\
\hline
\end{tabular}

Médias seguidas de mesma letra, na coluna, não diferem entre si pelo teste de Scott Knott a 5\%; ${ }^{1 /}$ Ingrediente ativo; ${ }^{2 /}$ Equivalente ácido, utilizado para o herbicida haloxyfop-methyl; ${ }^{3 /}$ Dias após a aplicação. 
Tabela 6 - Produtividade de grãos da cultura da soja após a aplicação de diferentes tratamentos herbicidas. Rio VerdeGO. 2006/2007

\begin{tabular}{|c|c|c|}
\hline \multirow[b]{2}{*}{ Tratamento } & Dose & \multirow{2}{*}{$\begin{array}{l}\text { Produtividade de } \\
\text { grãos } \\
\left(\mathrm{kg} \mathrm{ha}^{-1}\right)\end{array}$} \\
\hline & $\begin{array}{l}(\text { g i.a. })^{1 /} \text { ou } \\
\text { (e.a. })^{2 /} \mathrm{ha}^{-1}\end{array}$ & \\
\hline clethodim & 84 & $2.388 \mathrm{a}$ \\
\hline clethodim + quizalofop-p-ethyl & $48+40$ & $2.364 \mathrm{a}$ \\
\hline [clethodim + fenoxaprop-p-ethyl] & $50+50$ & $2.547 \mathrm{a}$ \\
\hline sethoxydim & 230 & $2.430 \mathrm{a}$ \\
\hline tepraloxydim & 100 & $2.205 \mathrm{a}$ \\
\hline fluazifop-p-butyl & 125 & $2.151 \mathrm{a}$ \\
\hline haloxyfop-methyl & 60 & $2.255 \mathrm{a}$ \\
\hline testemunha sem herbicida & - & $1.305 \mathrm{~b}$ \\
\hline $\mathrm{CV} \%$ & & 8,3 \\
\hline
\end{tabular}

Médias seguidas de mesma letra, na coluna, não diferem entre si pelo teste de Scott Knott a 5\%; ${ }^{1 /}$ Ingrediente ativo; ${ }^{2}$ Equivalente ácido, utilizado para o herbicida haloxyfop-methyl; ${ }^{3 /}$ Dias após a aplicação

Outra vantagem da utilização desta classe de herbicidas (inibidores da ACCase) é a alta seletividade desses compostos à cultura da soja. Durante a condução do experimento não foram detectados sintomas de injúria nas plantas da cultura em nenhum dos tratamentos herbicidas avaliados.

De todos os princípios ativos avaliados, constatou-se que haloxyfop-methyl apresenta o maior espectro de ação, considerando o universo das gramíneas avaliadas. Sua recomendação pode trazer maior segurança em casos em que não se dispõe de especialistas para a correta identificação das gramíneas infestantes.

\section{LITERATURA CITADA}

ASKEW, S. D.; SHAW, D. R.; STREET, J. E. Graminicide application timing influences red rice (Oryza sativa) control and seedhead reduction in soybean (Glycine max)

Leed Technol., v. 14, n. 1, p. 176-181, 2000.

BARROS, A. C.; MATOS, F. S. A.; NETTO, C. T. Avaliação de herbicidas no controle de plantas daninhas na cultura da soja. Planta Daninha, v. 10, n. 1/2, p. 45-49, 1992.

BURKE, I. C. et al. A seedling assay to screen aryloxyphenoxypropionic acid and cyclohexanedione resistance in johnsongrass (Sorghum halepense). Weed Technol., v. 20, n. 4, p. 950-955, 2006
CHERNICKY, J. P.; GOSSETT, B. J.; MURPHY, T. R. Factors influencing control of annual grasses with sethoxydim or RO13-8895. Weed Sci., v. 32, n. 2, p. 174-177, 1984

CRUZ, L. S. P. et al. Herbicidas de aplicação em pósemergência em amendoim: I. Controle de plantas daninhas e persistência no solo. Bragantia, v. 50, n. 1, p. 103-114, 1991

CHRISTOFFOLETI, P. J.; KEHDI, C. A.; CORTEZ, M. G Manejo da planta daninha Brachiaria plantaginea resistente aos herbicidas inibidores da ACCase. Planta Daninha, v. 19, n. 1, p. 61-66, 2001

DEER, J. F.; MONACO, T. J.; SHEETS, T. J. Response of three annual grasses to fluazifop. Weed Sci., v. 33, n. 5, p. 693-697, 1985.

DEFELICE, M. S. et al. Weed control in soybeans (Glycine max) with reduced rates of postemergence herbicides.

Weed Sci., v. 37, n. 3, p. 365-374, 1989

FLECK, N. G.; CANDEMIL, C. R. G. Interferência de plantas daninhas na cultura da soja. Ci. Rural, v. 25, n. 1, p. 27-32, 1995

FLECK, N. G.; CUNHA, M. M.; VARGAS, L. Dose reduzida de clethodim no controle de papuã na cultura da soja, em função da época de aplicação. Planta Daninha, v. 15, n. 1, p. $18-24,1997$

GAZZIERO, D. L. P. et al. Resistência da planta daninha capim-marmelada (Brachiaria plantaginea) aos herbicidas inibidores da enzima ACCase na cultura da soja.

Planta Daninha, v. 18, n. 1, p. 169-180, 2000.

GELMINE, G. A.; MATTOS, J. B. S.; NOVO, M. C. S. S Avaliação da eficiência do herbicida fenoxaprop-p-ethyl aplicado em pós-emergência da cultura da cebola. $\mathbf{R}$. Ecossistema, v. 26, n. 2, p. 135-138, 2001

HAGOOD JUNIOR., E. S. et al. Growth analysis of soybeans (Glycine max) in competition with velvetleaf (Abutilon theophrasti). Weed Sci., v. 28, n. 6, p. 729-734, 1980.

HARWOOD, J. L. Graminicides which inhibit lipid synthesis. Pest. Outlook, v. 10, n. 4, p. 154-158, 1999.

HOCHBERG, O.; SIBONY, M.; RUBIN, B. The response of ACCase-resistant Phalaris paradoxa populations involves two different target site mutations. Weed Res., v. 49, n. 1, p. $37-46,2009$

LINGENFELTER, D. D.; CURRAN, W. S. Effect of glyphosate and several ACCase-inhibitor herbicides on Wirestem Muhly (Muhlenbergia frondosa) control. Weed Technol., v. 21, n. 3, p. 732-738, 2007.

LÓPEZ-OVEJERO, R. F. et al. Suscetibilidade comparativa a herbicidas pós-emergentes de biótipos de Digitaria ciliaris resistente e suscetível aos inibidores da ACCase

Planta Daninha, v. 24, n. 4, p. 789-796, 2006. 
MARSHALL, G.; KIRKWOOD, R. C.; LEACH, G. E

Comparative studies on graminicide-resistant and susceptible biotypes of Eleusine indica. Weed Res., v. 34, n. 3, p. 177-185, 1994.

MEDRANO, C. et al. Evaluation of post-emergent herbicides for weed control on soybean (Glycine max (L.) Merr.) in the Maracaibo plateau. R. Facultad Agron. Universidad del Zuliav, v. 14, n. 1, p. 33-34, 1997.

NALEWAJA, J. D.; MATYSIAK, R.; SZELEZNIAK, E. F. Sethoxydim response to spray chemical properties and environment. Weed Technol., v. 8, n. 3, p. 591-597, 1994.
SILVA, A. A. et al. Controle de plantas daninhas. Brasília: Associação Brasileira de Educação Agrícola Superior, 1999. $260 \mathrm{p}$

SILVA, A. F. et al. Interferência de plantas daninhas em diferentes densidades no crescimento da soja. Planta Daninha, v. 27, n. 1, p. 75-84, 2009.

VIDAL, R. A. Ação dos herbicidas: absorção, translocação e metabolização. Porto Alegre: Evangraf, 2002. 89 p.

VIDAL, R. A. et al. Resistência de Eleusine indica aos inibidores de ACCase. Planta Daninha, v. 24, n. 1, p. 163-171, 2006. 